\title{
Influence of Acute Mental Arithmetic Stress on Taste and Pungency
}

\author{
Asuka SAWAI ${ }^{1}$, Takuma Motomura ${ }^{1}$, Tatsuhiro OSHIMA ${ }^{1}$, Shinya SAWAI ${ }^{2}$, \\ Tetsuya FujIKAWA ${ }^{3}$, Hitoshi FujII ${ }^{4}$, Yuichi BANNAI ${ }^{5}$, Yuichi TAKEDA ${ }^{6}$, \\ Masato $\mathrm{OHNO}^{7}$ and Osamu TochIKUBO ${ }^{8}$ \\ ${ }^{1}$ Department of Nutrition and Life Science, Kanagawa Institute of Technology, Atsugi 243-0292, Japan \\ ${ }^{2}$ Department of Applied Physics, National Defense Academy, Yokosuka 239-8686, Japan \\ ${ }^{3}$ Center for Health Service Sciences, Yokohama National University, Yokohama 240-8501, Japan \\ ${ }^{4}$ Department of Nursing, Mejiro University, Saitama 339-8501, Japan \\ ${ }^{5}$ Department of Information Media, Kanagawa Institute of Technology, Atsugi 243-0292, Japan \\ ${ }^{6}$ Center for Basic Education and Integrated Learning, Kanagawa Institute of Technology, \\ Atsugi 243-0292, Japan \\ ${ }^{7}$ National Institute of Technology, Yonago College, Yonago 683-8502, Japan \\ ${ }^{8}$ Yokohama City University, Yokohama 236-0004, Japan
}

(Received June 1, 2018)

\begin{abstract}
Summary Mental stress is a known risk factor for disease. This study investigated changes in sensations of taste and pungency before and after mental stress. Thirty healthy male university students rested for $20 \mathrm{~min}$, performed mental arithmetic tasks for $10 \mathrm{~min}$, and then underwent measurement of changes in their taste and ability to discern pungency. Taste was measured with the "Taste Disk ${ }^{\circledR}$," and pungency was measured by a filter-paper disc method using capsaicin solution. Subjects were not told the order of the reagent solutions used. To quantify pain sensation, a weak current applied to the central inner forearm skin by a Pain Vision ${ }^{\circledR}$ quantitative pain sensation analyzer was gradually increased. The degree of stress was measured by portable electrocardiography (ECG). During mental stress, the cognitive threshold of salty taste, sweet taste, and bitterness was significantly decreased, whereas the sensations of pungency and forearm skin pain were increased and showed significant correlation. Based on sympathetic nerve activity analyzed with the ECG, the subjects were divided into the mental stress group and non-mental stress group. The mental stress group experienced an increase in the pungency threshold and sensation of forearm skin pain with significantly high correlations obtained, whereas no correlation was found between these factors in the non-mental stress group. Acute mental stress increased the sensitivity to taste but decreased the sensitivity to the sensation of pungency on the tongue and pain on the skin. Sympathetic activity activated by stress may affect taste and the sensation of pungency.
\end{abstract}

Key Words taste, capsaicin, pungency, hot, mental stress, sympathetic nerve activity, current

The number of patients with lifestyle diseases (1), psychiatric diseases (2), and inflammatory and irritable bowel diseases (3) is increasing in developed countries, and the involvement of mental stress has been noted in these diseases $(3,4)$. In Japan, efforts are also being made to require companies to manage the mental stress of workers (5). Such social situations have led to an increase in academic research on stress in recent years.

Epidemiological studies have mainly reported that patients with hypertension (6), obese people (7), depressed patients $(8)$ and those thought to undergo long-term stress (9) experience abnormalities in appetite and taste. For example, in hypertension with salt sensitivity, a decrease in the ability to discern a salty taste due to a high salt intake habit is suggested to be a factor leading to an increase in blood pressure (6). In addi-

E-mail: asawai@bio.kanagawa-it.ac.jp tion, taste disorder in depression due to the exhaustion of neurotransmitters such as serotonin and adrenaline, the increased demand for zinc, which is a synthetic element of these neurotransmitters, and a decrease in the dietary intake of nutrients due to decreased appetite are also considered to be involved ( 8 ). In the case of persistent stress, cortisol is also secreted in addition to adrenaline and noradrenaline, which are enhanced at the time of rapid stress (9).

In recent years, receptors for noradrenaline and serotonin have also been found in the taste buds (10). Regarding the relation of stress with dietary intake, although a few studies have reported on changes in taste, with diverse results $(7-9,11-13)$, to our knowledge, there are hardly any reports of the effects of mental stress on the sensitivity to pungency.

Adrenaline and noradrenaline are also known to affect the central nervous system and to alleviate the 


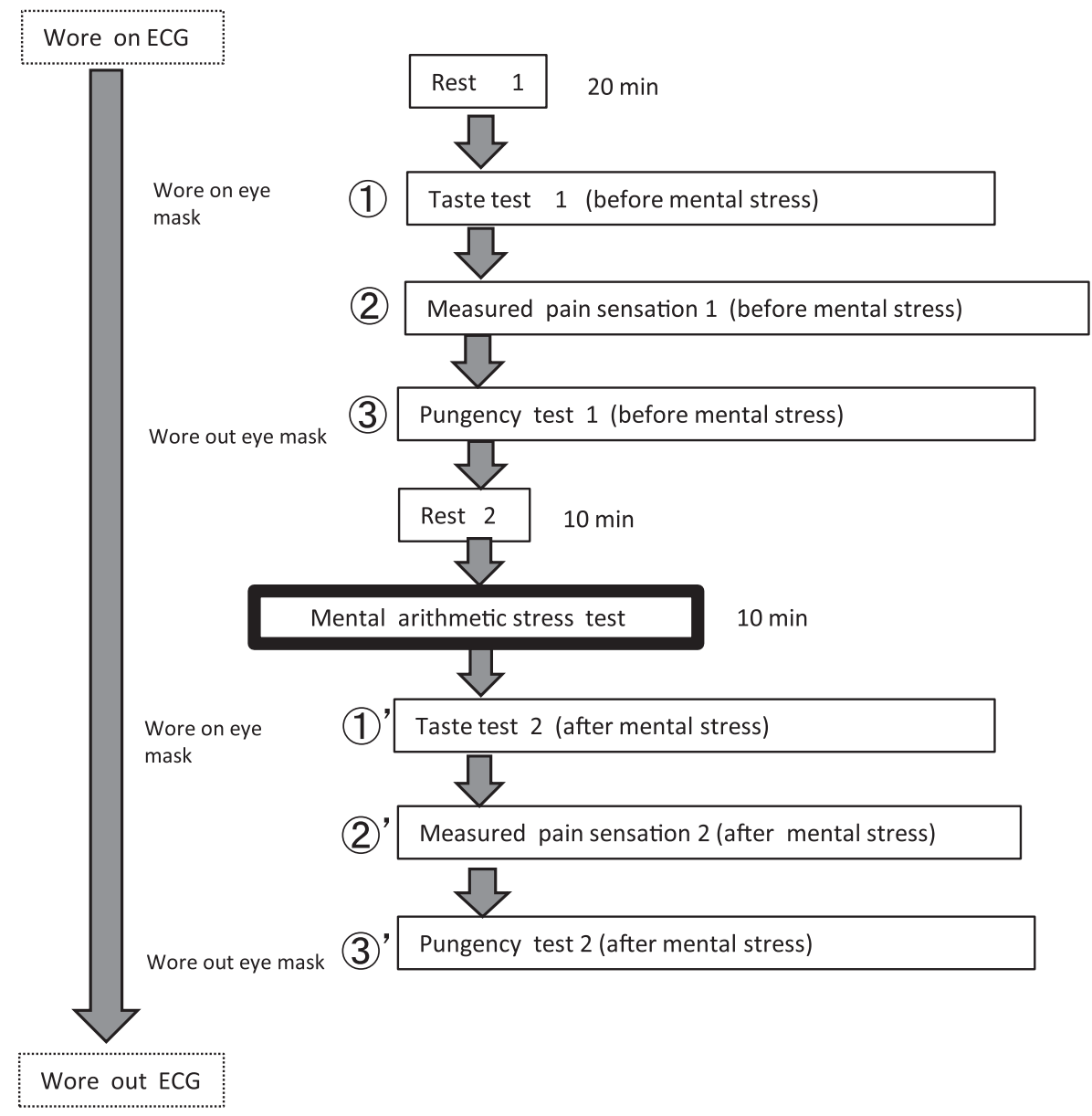

Fig. 1. Experimental protocol.

sensation of pain (14). The pain stimulus is sensed by nociceptors, the stimulation is brought to the forefront, and it is recognized by the cerebrum as pain through sympathetic mediation in the spinal cord. Depending on the magnitude of the pain, a reward system substance is secreted that relaxes the sensation of pain and pleasure. Various substances cause the body to feel pain, and pungent ingredients contained in foods and spices are one of them (14). Pungent taste is experienced as a pain sensation, not taste, as it causes pain, perspiration, fever, and psychological aspects such as pleasure associated with the sensation of pain (14). Red pepper is one of the spices used in various countries in Asia, and it has been used in meals in various ways in Japan in recent years (15). Capsaicin, the main ingredient in hot chili peppers, stimulates the temperature sensation through transient receptor potential cation channel subfamily $V$ member 1 (TRPV1) and induces a sensation of heat (16). It also highly influences physiological functions such as increasing body heat production even when a small amount is ingested orally (17). Red pepper and capsaicin help to expend body fat (17) and are used as analgesics (18) to aid in health care. However, these so-called spices including chilies are highly irritating to the gastrointestinal mucosal tissue (19-21), so as stimulants in colorectal diseases, these contents need to be considered depending on the symptoms.
Therefore, in this study, we investigated both the effect of stress on taste and pungency in subjects under a mental load and the effect of mental stress on pain sensation because pungency is perceived as pain.

\section{METHODS}

Subjects. The subjects of this study were 30 male university student volunteers (average age 21.07 $\pm 1.31 \mathrm{y}$ ). Subjects were selected according to the following criteria: 1) body mass index (BMI) from 18.5 to $24 \mathrm{~kg} / \mathrm{m}^{2}$, 2) moderate resistance to pungency as they do not eat pungent foods every day, but they do not dislike pungent foods, 3) no diseases of the cardiovascular system or gastrointestinal tract, and 4) no routine exposure to strong stress conditions. The average height of the subjects was $171.8 \pm 6.5 \mathrm{~cm}$, the average weight was $63.8 \pm 10.0 \mathrm{~kg}$, and the average BMI was $21.6 \pm 2.7 \mathrm{~kg} / \mathrm{m}^{2}$.

Measurements. The subjects wore electrocardiography (ECG) electrodes (disposable monitoring electrode L bead load; Nihon Kohden Industry Co., Ltd.) attached to an ECG monitoring system (TM2425-ECG; A \& D Company) (22). Subjects were examined during weekdays from 9 am to 12 am while being kept fasted. To avoid effects from differences in hunger state and taste sensation of the subjects' ingested breakfast on the experimental results, we required the subjects to finish breakfast $3 \mathrm{~h}$ before the experiment, not to ingest any 
Table 1. Changes in cardiovascular indices caused by mental stress.

\begin{tabular}{|c|c|c|c|c|c|}
\hline & $\mathrm{SBP}(\mathrm{mmHg})$ & $\mathrm{DBP}(\mathrm{mmHg})$ & HR (beats/min) & $\begin{array}{c}\text { Parasympathetic } \\
\text { nerve activity }\left(\mathrm{ms}^{2}\right)\end{array}$ & $\begin{array}{c}\text { Sympathetic } \\
\text { nerve activity (ratio) }\end{array}$ \\
\hline Rest 1 & $115.7 \pm 24$ & $66.4 \pm 15$ & $86.4 \pm 26$ & $28.7 \pm 14.1$ & $2.6 \pm 0.9$ \\
\hline $\begin{array}{l}\text { Taste test } \\
\text { (before mental stress test) }\end{array}$ & $120.1 \pm 20$ & $67.7 \pm 13$ & $81.7 \pm 21$ & $30.4 \pm 15.6$ & $2.4 \pm 0.6$ \\
\hline $\begin{array}{l}\text { Measured pain sensation } \\
\quad \text { (before mental stress tset) }\end{array}$ & $123.6 \pm 21$ & $73.3 \pm 1.4$ & $85.6 \pm 20$ & $29.1 \pm 13.8$ & $2.2 \pm 0.6$ \\
\hline $\begin{array}{l}\text { Pungency test } \\
\text { (before mental stress test) }\end{array}$ & $127.1 \pm 23$ & $74.5 \pm 18$ & $84.6 \pm 24$ & $28.2 \pm 15.3$ & $2.2 \pm 0.6$ \\
\hline Rest 2 & $126.6 \pm 22$ & $74.4 \pm 23$ & $80.1 \pm 16$ & $28.7 \pm 13.4$ & $2.1 \pm 0.6$ \\
\hline Mental arithmetic stress test & $145.7 \pm 26 \mathrm{ab}$ & $74.2 \pm 19$ a & $88.5 \pm 23 b$ & $26.8 \pm 13.9 \mathrm{ab}$ & $2.9 \pm 0.8 \mathrm{ab}$ \\
\hline $\begin{array}{l}\text { Taste test } \\
\quad \text { (after mental stress test) }\end{array}$ & $140.8 \pm 24 \mathrm{c}$ & $74.0 \pm 15$ & $79.2 \pm 19$ & $28.1 \pm 15.8$ & $2.8 \pm 0.7 \mathrm{c}$ \\
\hline $\begin{array}{l}\text { Measured pain sensation } \\
\quad \text { (after mental stress test) }\end{array}$ & $139 \pm 23 c$ & $70.2 \pm 15$ & $83.3 \pm 21$ & $30.4 \pm 27.7 \mathrm{c}$ & $2.7 \pm 0.6 \mathrm{c}$ \\
\hline $\begin{array}{l}\text { Pungency test } \\
\quad \text { (after mental stress test) }\end{array}$ & $138.3 \pm 22 \mathrm{c}$ & $66.2 \pm 19$ & $82.3 \pm 16$ & $27.4 \pm 18.1$ & $2.6 \pm 0.6 \mathrm{c}$ \\
\hline
\end{tabular}

The values are expressed as the mean \pm SD. SBP, systolic blood pressure; DBP, diastolic blood pressure; HR, heart rate. Comparison by $t$-test. $p<0.05$. a, vs. rest 1 ; b, vs. rest 2 ; c, vs. each rest period (before mental stress test).

stimulants or taste anything, and to drink only water. On the day of the experiment, subjects were confirmed not to have taken breakfast or food and drink other than water and not to have smoked.

The protocol is shown in Fig. 1. The subjects rinsed the inside of their mouth with water and then sat at rest. Thereafter, ECG of each subject was measured at rest for $20 \mathrm{~min}$ (rest 1). Then, the tests of taste, the pain sensation and pungency were conducted while with eyes masked. The pain sensation of the subjects was measured 3 times at rest with a quantitative pain analysis device (Pain Vision ${ }^{\circledR}$; NIPRO Co., Ltd.) through electrodes (23) applied to the central brachial surface of the left forearm. Then, the eye mask was removed and ECG of each subject was measured at rest for $10 \mathrm{~min}$ (rest 2 ) and the mental arithmetic test was done for $10 \mathrm{~min}$. Then, tests of taste, the pain sensation and pungency were conducted while an eye mask was worn.

Mental arithmetic test. As a stress load, a mental calculation task was presented on a PC monitor, and the subjects entered answers to this mental stress load for 10 min (24). The mental arithmetic stress test involved serial subtraction of 17 from 8,500 ; thus, subjects progressively calculated $8,500-17,8,483-17$, and so on. Immediately after this stress load, tests of taste and pungency were conducted again.

Monitoring of blood pressure, heart rate, and sympathetic activity. ECG and heart rate (HR) were recorded beatto-beat during rest and the mental stress tests. Blood pressure was measured every 3 min during rest and the tests. Low-frequency (LF; 0.04-0.15 Hz) and high-frequency (HF; 0.15-0.40 Hz) components of the ECG RR interval power spectrum derived from the TM2425-ECG recorder were analyzed. The LF/HF ratio was regarded as an index of sympathetic activity. Mean HR, mean sys- tolic blood pressure (SBP) and diastolic blood pressure (DBP), and mean LF/HF ratio were calculated for the initial resting period and during the stress tests $(25,26)$.

Taste test. The taste test was conduct using the filterpaper disc method (24). The filter papers were placed in a $37^{\circ} \mathrm{C}$ water bath of liquids with various percent concentrations of four tastes: sweet: $0.3,2.5,10,20$, and 80\%; sour: $0.0,0.2,2,4$, and 8\%; salty: $0.3,1.25,5$, 10, and 20\%; and bitter: 0.001, 0.02, 0.1, 0.5, and 4\% (Taste Disk ${ }^{\circledR}$, Sanwa Chemistry Research Lab., Inc.) (27). The subjects wore an eye mask during the taste examinations and pungency examinations to ensure accurate examination. The eye mask was removed during rest 2 and the mental arithmetic stress test. The subjects replied to the question of whether they could taste the filter paper after it was placed on the tip of their tongue for $30 \mathrm{~s}$. The subjects washed their mouth with water after each filter-paper disc was removed.

Pungency test. The pungency test was also conducted using the filter-paper disc method. During the pungency testing, the patient was blinded to the order of application of the pungent solutions and to questions about the pungency. In accordance with prior studies (28), the capsaicin solution (capsicum extract No. 1lco7jo45; Alps Pharmaceutical Industry Co., Ltd.) was diluted to the following concentrations in pure water (capsaicin aqueous solution): $0.1,0.2,0.3,0.6,1,1.9,3.2,5.6$, $10,20,30,60,100,190,320$, and 560 ppm. Measurement of the sensation of pungency in the subjects continued up to the maximum concentration that each subject could handle (maximum pungency sensitivity).

Examination of pain sensation of the skin. While blindfolded in the resting state, each subject experienced gradually increased weak electric current emitted from the Pain Vision ${ }^{\circledR}$ device, and the current values at the 

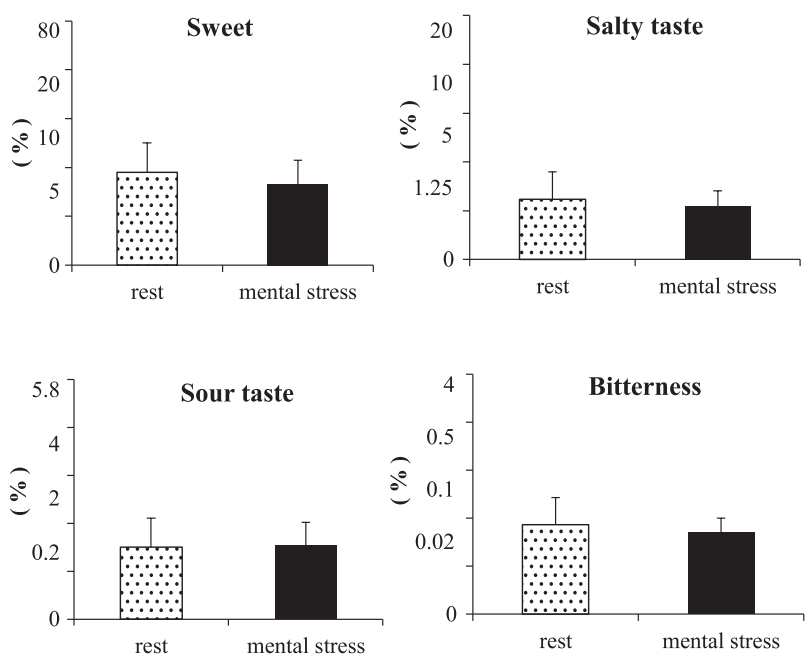

Fig. 2. Comparison of minimum threshold tastes before and after the mental stress test. The values on the $Y$ axis indicate the concentration of the taste that was discerned. Values are expressed as the mean \pm SD. Comparison by Wilcoxon signed-rank test. ${ }^{*} p<0.05$.

point where the current was first discerned (minimum sensing threshold) and at the point where pain was first felt (cognitive threshold) were measured. The protocol is shown in Fig. 1.

Statistical analysis. Values are expressed as mean \pm SD. The threshold of taste before and after the stress load and the threshold of pungency were assessed with the Wilcoxon rank sum test. The detection of forearm pain, the pain threshold of the forearm, HR, SBP, HF, and LF/ HF were assessed with a $t$-test. Comparison of pungency threshold and forearm pain was conducted with Spearman correlation analysis. The significance level was set at $p<0.05$.

Ethics committee. This research was approved by the Kanagawa Institute of Technology University (approval no. 20171212-22). All subjects were provided detailed information on the experiment and gave their written informed consent. This study was conducted in accordance with the Declaration of Helsinki.

\section{RESULTS}

The results of the cardiovascular function measurements during the mental arithmetic stress test are shown in Table 1 . The results of statistical analysis are indicated by the lowercase letters to the right of the values measured during or after mental arithmetic stress loading. The details of this notation are as follows: the results during mental arithmetic stress loading were compared with both "rest 1" and "rest 2," and those that showed a significant difference with "rest 1" are identified with the letter "a." Those that showed a significant difference with "rest 2" are identified with "b." For the taste test, the pain sensation test, and the pungency test, the value at rest corresponding to each measurement was compared with the value after stress loading. If a significant difference was observed in the test results, it was identified with the letter "c." For example,
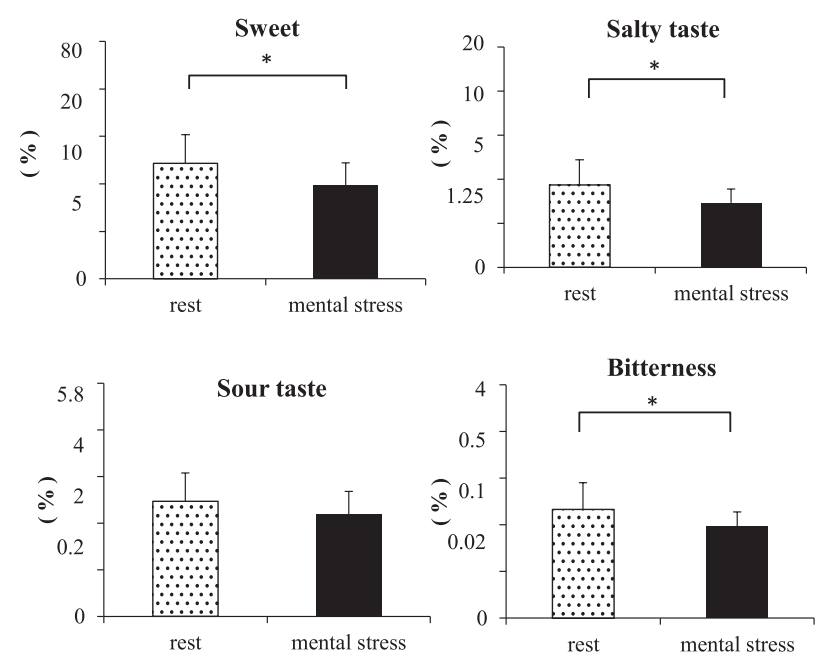

Fig. 3. Comparison of the concentrations of the recognized threshold tastes before and after the mental stress test. Values are expressed as the mean \pm SD. Comparison by Wilcoxon signed-rank test. ${ }^{*} p<0.05$.

the systolic blood pressure data were compared between before and after the mental arithmetic loads in the taste test and are marked with a "c." Items not showing a significant difference were not identified with letters after the mental stress calculation.

Each measured value of HR, SBP, DBP, and sympathetic nervous activity was significantly higher during mental arithmetic than during rest (rest 1 or rest 2) $(p<0.05)$. The value for parasympathetic nervous activity during mental arithmetic was significantly lower than that during rest (both rest 1 and rest 2$)(p<0.05)$. Values of SBP and sympathetic activity measured during the taste test, pain sensation test, and pungency test were significantly higher $(p<0.05)$ after the mental arithmetic stress load than before it.

The results of the taste test are shown in Fig. 2. The mean values showed a small decrease in the sensing threshold for discerning sweet, salty, sour, and bitter tastes after the mental stress, but the differences were not significant between before and after the mental stress test. The mean values of the cognitive threshold for sweet, salty, sour, and bitter tastes also showed a decrease after the mental stress, and those differences were significant $(p<0.05)$ (Fig. 3). The mean maximum sensitivities to pungency concentrations were significantly higher after mental stress than those measured before mental stress $(p<0.05)$ (Fig. 4). The degree of pain felt in the forearm skin during mental stress was significantly higher than that felt at rest. Furthermore, on the basis of a central value of 0.3 of the increase (between rest 1 and during the mental arithmetic stress test) in sympathetic nerve activity (determined by subtracting the rest value from the mental stress value), the subjects were divided into two groups, the mental stress group and non-mental stress group, and compared. There was a significant positive correlation between the degree of increase in sensitivity to the maximum pun- 

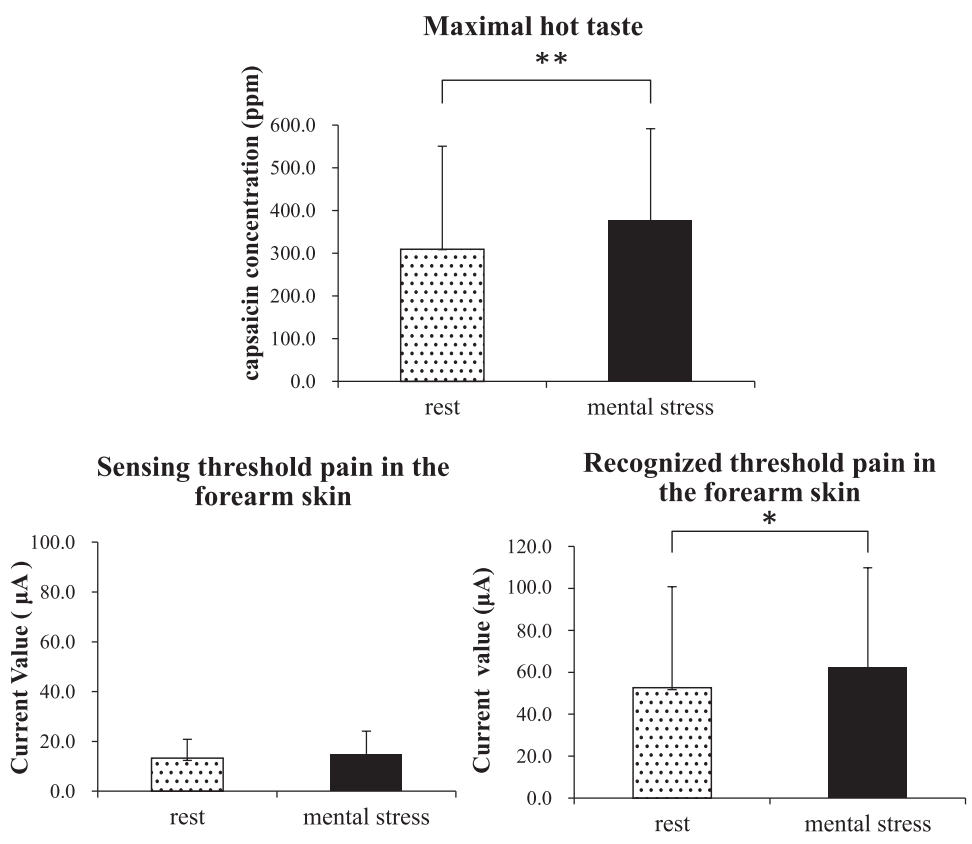

Fig. 4. Comparison of the pungency of the maximally sensitive concentration before and after the mental stress test. Comparison of the electrical stimulation value of the pain sensitivity in the forearm skin before and after the mental stress test. Values are expressed as the mean \pm SD. Comparison by $t$-test. ${ }^{*} p<0.05$, ${ }^{* *} p<0.01$.

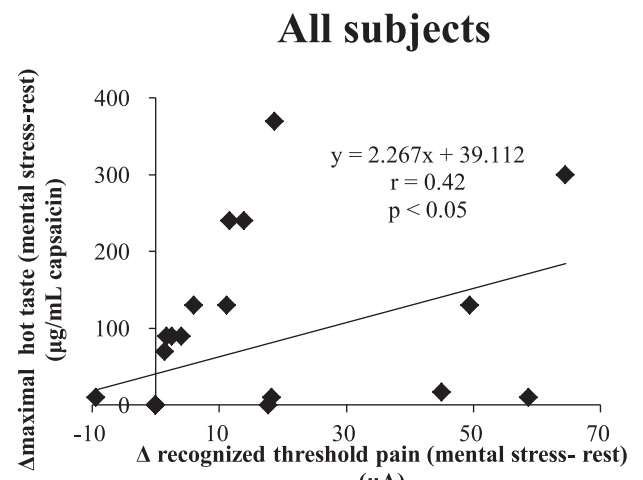

$(\mu \mathrm{A})$
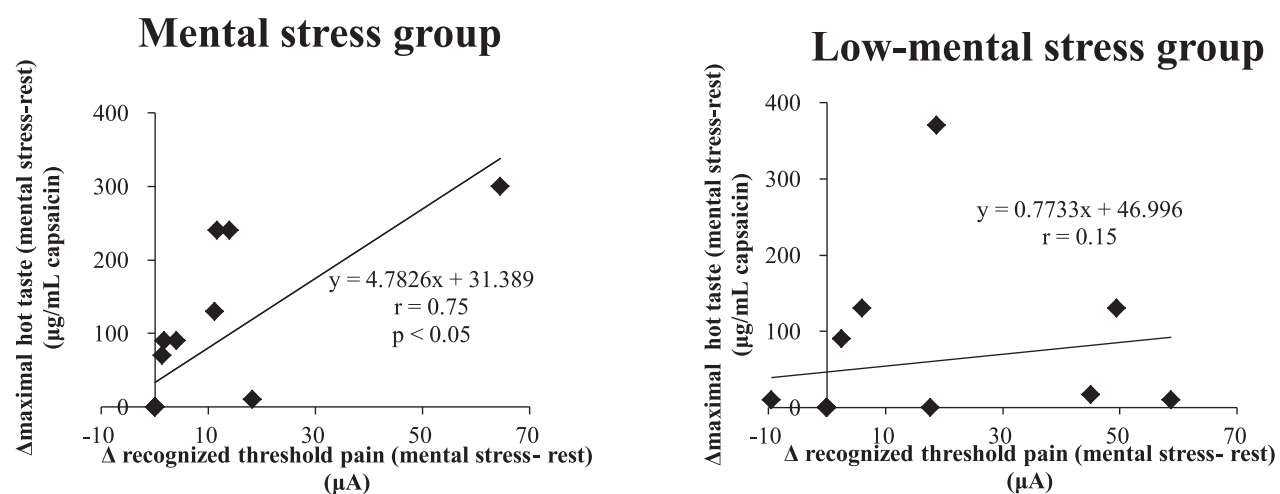

Fig. 5. Relation between the degree of pain and the pain-compatible electrical stimulation according to capsaicin intake. The comparison of pungency threshold and forearm pain was conducted by Spearman correlation analysis. The significance level was set at $* p<0.05$. 
gency concentration and the degree of increase in pain current in the mental stress group, but there was no correlation in the low-mental stress group (Fig. 5).

\section{DISCUSSION}

In this study, we investigated whether sensations of taste and pungency are affected by mental stress. The mental stress task used experimentally to create a stress environment is a mental arithmetic task that is often used in research (29). As indicated in Table 1, both the subjects' cardiovascular functions (blood pressure and HR) and sympathetic nerve activity increased while performing the mental arithmetic task. The fluctuation of autonomic nervous activity during stress loading was confirmed by an increase of 0.5 in the LF/HF ratio reflecting sympathetic nerve activity and a decrease of $10 \mathrm{~ms}^{2}$ in average parasympathetic activity, both of which showed significant differences during the stress load. This suggested that the subjects experienced stress that changes autonomic nervous activity. Although it is presumed that a subject's experience of stress and autonomic nerve variability depends on the susceptibility of the individual to stress and the influence on the autonomic nerves, the fluctuation of autonomic nervous activity after stress was comparable to that noted in a previous report (24). In addition, although there were significant differences in the mental stress (between rest 1 and during the mental arithmetic stress test) of the subjects, as Table 1 shows, the range of fluctuation was large, and individual differences in susceptibility to stress were great. Incidentally, because controlling respiration itself may be a stress, respiratory control was not done in the present study. Measurements of ECG for which breathing is not regulated in this way are performed in many clinical studies (22, 24-26). Furthermore, analysis of autonomic nerve activity is based on the low probability of occurrence of respiratory arrhythmia due to mild stress loading in healthy young people and automatic elimination by the analysis device when an arrhythmia does occur.

As indices of taste, the "detection threshold" indicates a concentration that does not lead to discrimination of taste although there is an uncomfortable feeling, but the "cognitive threshold" indicates a concentration at which taste can be discriminated for the first time. A high threshold means that the sensation of taste is low (dull), and a low threshold means that the sense of taste is high (sharp). The mean values of the cognitive thresholds of the four basic tastes (i.e., sweet, salty, sour, and bitter) were all decreased after the mental stress, and sweet, salty, and bitter tastes were significantly decreased under the influence of the mental stress load. In previous studies, Ileri-Gurel et al. examined the change in sweetness and salty taste before and after the Stroop color words test and found that the threshold for both tastes decreased (i.e., the tastes became sensitized) (7). Heath et al. investigated the four tastes in subjects taking a dose of noradrenaline or serotonin as a formulation rather than when it is secreted during stress sensitivity in a direct stress test. After the subjects took serotonin, the thresholds of sweetness and bitterness decreased (i.e., the sensitization of sweetness and bitterness was activated). After the subjects took noradrenaline, the threshold of bitterness decreased (i.e., the experience of bitterness was sensitized) (10). Rai and Kaur (12) indicated that the response to bitterness, sweetness, and sourness became quicker and that acidity may be due to the increased $\mathrm{pH}$ caused by the increase in the amount of $\mathrm{HCl}$ in saliva, as evidenced by the bitter taste of mouse saliva due to proline-rich proteins following administration of a $\beta$ adrenergic. Therefore, they estimated that the increase in bitterness is due to transporting substances in saliva resulting from stress (12). The salty taste results were different between Ileri-Gurel et al. and Heath et al. Our study results were similar to those of Heath et al. and showed a decrease in threshold due to stress (i.e., taste sensitization). The burden of stress on humans and stress due to medication are not necessarily the same, so further studies are awaited.

In the in vitro research, recent studies have shown that taste receptor cells respond to noradrenaline (10) and that the action of noradrenalin enhances the response of gustatory nerves (10). As in the present study, it is considered that an increase in cardiovascular function observed by accelerated sympathetic nervous activity is mainly due to adrenaline and noradrenaline released at the time of an immediate stress burden. This therefore suggests that the increase in the cognitive threshold of taste was caused by these nerves and implies that this is due to activation in taste cells of transmitter substances in the sympathetic nervous system by mental stress.

In contrast, there have been reports in epidemiological studies that people exposed to long-term stress (9), depressed patients (12), and obese people (7) have a weak sense of taste. In addition, the four tastes were investigated during relatively short periods of mental stress ( 4 min of public speaking, 8 min of mental arithmetic, and $90 \mathrm{~s}$ of cold stimulation by ice on the fingertips). A significant decrease in the taste of sweetness was observed, and the relationship with the cortisol value measured at the same time was described (9). According to the reports (9), cortisol is stated as increased at mental stress. In the case of persistent stress, cortisol is also secreted in addition to adrenaline and noradrenaline, which are enhanced at the time of rapid stress (9). In recent years, cortisol receptor expression was confirmed in confined rat taste cells (27). Because we did not measure cortisol in our study, we do not know the details of its secretion. Of concern, when considering stress load time and circulatory dynamics, a stress that would be influenced by cortisol would be overly long and the load would be excessive. In the results of previous studies (7-13), no consensus as to the effect of psychological stress on cognitive threshold has been reached. In addition to the lack of research from the beginning, the kind of mental stress, the load condition, and the inspection items are not standardized. Although the basic neuroscience of the stress response is currently well understood, our understanding of the relation between this system and taste is limited. Thus, further investigation is 
needed from the viewpoints of molecular biology, physiology, and nutrition.

In the test of pungency, the threshold increased after mental stress, indicating that the sensation of pungency was decreased. The capsaicin used as a pungent substance is a kind of spice that modifies the taste of food. Capsaicin is not sensed by taste bud receptors but is sensed at one of the TRPV member pain sensors, the capsaicin receptor (TRPV1) (16). Thus, because pungency is recognized as a sense of pain, we investigated the partial reaction to pain by the full body due to stress by evaluating skin pain using low-current stimulation.

After mental stress, skin pain decreased, and its blunting was particularly noticeable in those subjects with increased sympathetic nerve activity. To our knowledge, there are almost no reports of the influence of stress on the sensation of pungency. However, there are reports of skin pain sensation caused by stress and capsaicin applied to the skin instead of the tongue, so we compared our research results to these reports. One report noted that the pain of capsaicin coating the skin decreased after an acute mental stress test (30). Another report showed that the susceptibility to skin current decreased after the performance of a mental arithmetic task similar to that of the present study (31).

Pain substances are sensed at the receptors of pain sensation. The signals enter the spinal cord via sensory nerves, are transferred to neurons in the spinal cord, pass through the medulla oblongata, and are recognized as pain in the cerebral perception area (29-31). Besides sensory nerves in the spinal cord, sympathetic nerves and parasympathetic nerves are also present and have changeable neurons. Therefore, it is possible that the sensory nerve can receive the action of noradrenaline secreted from the stress-activated sympathetic nerve. In addition, it is known from various studies that noradrenaline and adrenaline have pain-alleviating actions and are actually neurotransmitters of a low-level suppression system that suppresses upper-brain dominance of the spinal cord (29-32). In the process of cognition of pain in the cerebrum, the sensory nerve also receives modifications such as serotonin, $\beta$-endorphin, GABA, etc., secreted from the brain stem and pituitary and is recognized in a relaxed state according to the degree of stimulation (29-31). In our study, because we did not measure substances such as $\beta$-endorphin and cortisol, the relationship with these substances that regulate pain is unknown, so the mechanism of pain recognition and capsaicin application on the tongue and pain during stress is unknown. As a result of a previous study, the significant correlation between the degree of increase of sympathetic nerve activity and activity of capsaicin concentration was known. So, at least, the increase in sympathetic nerve activity due to the short period of mental stress affected pungent sensation in our study.

The results of both the taste detection threshold and minimum skin current detection threshold were less affected by mental stress. The detection threshold of taste refers to "the point at which a taste is unknown, but some sense of discomfort is felt on the tongue," and the minimum current detection threshold of pain sensation also indicates the "sensed point." In other words, although both thresholds are incompatible, it means that a taste or pain sensation has not been discerned yet. There are many papers on taste testing that do not describe results related to the detection threshold (7-13), but the studies that do mention it are those of cross-sectional research comparing different people. For example, a report that examined aging and taste showed that the detection threshold and the cognitive threshold become dull as age increases (32). In addition, in reports comparing healthy subjects with those with dementia, the patients with dementia experience an increase in the cognitive threshold from the beginning, but the detection threshold increases later (33)

Although there are a few cases where both the detection threshold value and the cognition threshold value are described in the load test on the same subject, the papers state that a fluctuation in the cognitive threshold occurs but that no change occurs in the detection threshold (10). The reason is that in the case of feeling a sensation before cognition, due to the subject's belief, the sensation is regarded as another taste and it is difficult to obtain accurate information. There are descriptions in the literature that compare pain sensations with other causes of pain; for example, the results of survey of pain sensation from temperature stimulation were reported that made comparisons across different people in crosssectional research (34). The load test results of taste in the same person do not seem to change very much. Thus, because there is little information on the detection threshold, it is difficult to understand this threshold in detail, but it may be different among individuals and variation within an individual may be small. In other words, it may be hard for noradrenaline, etc., secreted at the time of stress to affect the threshold in the state before a person is susceptible to taste and pain sensation even under stress load, but the details will be revealed in future research.

However, it is also known that cortisol secreted by the adrenal glands due to long-term stress is secreted in response to particularly strong pain.

The influence of and the resistance to the taste threshold can differ depending on the type and degree of stress.

When the results of both taste and pungent sensation were integrated, similarity was observed in the detection threshold or minimum detection threshold, but the cognitive threshold was different in both reactions. The following four points were inferred for the different reactions of the cognitive threshold. The first is a conjecture about the action pathway of noradrenaline (14). The taste buds comprise two-thirds of the tongue from the tip, which is innervated by the lingual nerve, to the glossopharyngeal nerve, which innervates the back portion (14). The taste test was performed on the tip of the tongue, so lingual innervation was considered to be involved. Taste substances pass through the taste buds, then from the tongue nerve through the tympanic nerve to the facial nerve, and neurons are changed in the 
medulla oblongata $(7-10)$. The second neuron route is from the solitary nucleus of the medulla oblongus to the thalamus, and the third neuron is thought to be from the hypothalamus to the cerebral cortical taste field (27) although there are still other complicated pathways in the medulla oblongata and hypothalamus (27). In sympathetic neurons of the upper part of the spinal cord, acetylcholine is secreted in the anterior fibers, and noradrenaline is released from the end of the descending fibers of the nerve cells (14). In other words, considering the transmission path of the nerve, the sensory nerve that conveys the feeling of pain transfers neurons in the spinal cord, so the effect of noradrenaline secreted from the sympathetic nerve endings is stronger than that of the nerve conveying the sensation of the taste transferred to neurons in the medulla oblongata, and thus the pain sensation may be easier to receive. The second point concerns effects other than those from the sympathetic nerve. Pain may also be due to the involvement of these other effects as it is known that pain can be relieved by serotonin or $\beta$-endorphin, in addition to noradrenaline $(18,31,33)$. As a third point, as confirmed in cultured taste cells (10), it is possible that tissues other than nerves are activated by stress. Fourth is that the target of noradrenaline caused by activation of the sympathetic nerve in response to short-term stress differs between the sensory nerve transmitting pain and the nerve transmitting taste, but the details are unclear. These many unclear details are left to future studies.

There are some individual differences in pungency (pain) between the kinds of receptors, and the action of mitigating substances in the neurotransmission pathway is assumed (35-38). Moreover, when people eat pungent foods little by little from childhood, they build tolerance to and get used to these foods (2). Changes in tolerance and resistance to nerve reactions due to repeated ingestion of capsaicin were observed in animal experiments (18). Therefore, to improve the accuracy of the present research, the subjects were limited to those who were able to taste all of the test solution concentrations prepared in advance and did not have daily extremes of pungent taste. The present results and previous literature suggest that relaxation occurs in the recognition of pungency (pain) in the brain during mental stress. We thought that this relaxation is due to the influence of sympathetic nervous activity accelerated by the mental stress load in the spinal cord. It was interesting that the influence on taste and pain sensation differed due to an increase in sympathetic nerve activity caused by an immediate short-term mental stress. The relation between the sense of taste and food intake is important because it implies the morbidity of various diseases and their possible prevention.

The present study has limitations. First, the mental arithmetic load used was short term and probably induced only a small stress. The mental stresses of social life are long term and can be severe. It will be necessary to pursue further study of changes in the sensations of taste and pungency under such long-term and variable stresses. Second, because the electrode used by the
Pain Vision ${ }^{\circledR}$ cannot be applied to the tongue, the pain threshold was measured on the skin of the inside forearm, which is the electrode location designated for the device.

The acute mental arithmetic load caused a decrease in the taste threshold and an increase in the pungency threshold. Subjects whose sensation of pungency was reduced by stress also experienced decreased pain sensation of the skin, and the correlation between them was significant. This suggested that the sensations of taste and pungency may be adjusted by substances activated by mental stress. Regarding the relation between sensation and stress, which is still being clarified in molecular biology, the results of this study may provide information that supports its contents from the viewpoint of human physiological experiments. Because many unclear points remain regarding the influence of mental stress on the sensations of taste and pungency and the current information is limited, further study will be required in the future.

\section{REFERENCES}

1) Rastam S, Al Ali R, Maziak W, Mzayek F, Fouad FM, O'Flaherty M, Capewell S. 2012. Explaining the increase in coronary heart disease mortality in Syria between 1996 and 2006. BMC Public Health 12: 754.

2) Demyttenaere K, Bruffaerts R, Posada-Villa J, Gasquet I, Kovess V, Lepine JP, Angermeyer MC, Bernert S, de Girolamo G, Morosini P, Polidori G, Kikkawa T, Kawakami N, Ono Y, Takeshima T, Uda H, Karam EG, Fayyad JA, Karam AN, Mneimneh ZN, Medina-Mora ME, Borges G, Lara C, de Graaf R, Ormel J, Gureje O, Shen Y, Huang Y, Zhang M, Alonso J, Haro JM, Vilagut G, Bromet EJ, Gluzman S, Webb C, Kessler RC, Merikangas KR, Anthony JC, Von Korff MR, Wang PS, Brugha TS, Aguilar-Gaxiola S, Lee S, Heeringa S, Pennell BE, Zaslavsky AM, Ustun TB, Chatterji S; WHO World Mental Health Survey Consortium. 2004. Prevalence, severity, and unmet need for treatment of mental disorders in the World Health Organization World Mental Health Surveys. JAMA 291: 2581-2590.

3) Ng SC, Shi HY, Hamidi N, Underwood FE, Tang W, Benchimol EI, Panaccione R, Ghosh S, Wu JCY, Chan FKL, Sung JJY, Kaplan GG. 2018. Worldwide incidence and prevalence of inflammatory bowel disease in the 21st century: a systematic review of population-based studies. Lancet 390: 2769-2778.

4) Bannai A, Tamakoshi A. 2014. The association between long working hours and health: a systematic review of epidemiological evidence. Scand J Work Environ Health 40: $5-18$.

5) Ito S, Fujita S, Seto K, Kitazawa T, Matsumoto K, Hasegawa T. 2014. Occupational stress among healthcare workers in Japan. Work 49: 225-234.

6) Stolarz K, Staessen JA, Kawecka-Jaszcz K, Brand E, Bianchi G, Kuznetsova T, Tikhonoff V, Thijs L, Reineke T, Babeanu S, Casiglia E, Fagard R, Filipovský J, Peleska J, Nikitin Y, Struijker-Boudier H, Grodzicki T; European Project On Genes in Hypertension (EPOGH) Investigators. 2004. Genetic variation in CYP11B2 and AT1R influences heart rate variability conditional on sodium excretion. Hypertension 44: 156-162.

7) Ileri-Gurel E, Pehlivanoglu B, Dogan M. 2013. Effect of 
acute stress on taste perception: in relation with baseline anxiety level and body weight. Chem Senses 38: 27-34.

8) Newcomer JW, Selke G, Melson AK, Gross J, Vogler GP, Dagogo-Jack S. 1998. Dose-dependent cortisol-induced increases in plasma leptin concentration in healthy humans. Arch Gen Psychiatry 55: 995-1000.

9) Al'Absi M, Nakajima M, Hooker S, Wittmers L, Cragin T. 2012. Exposure to acute stress is associated with attenuated sweet taste. Psychophysiology 49: 96-103.

10) Heath TP, Melichar JK, Nutt DJ, Donaldson LF. 2006. Human taste thresholds are modulated by serotonin and noradrenaline. J Neurosci 26: 12664-12671.

11) Nakagawa M, Mizuma K, Inui T. 1996. Changes in taste perception following mental or physical stress. Chem Senses 21: 195-200.

12) Rai B, Kaur J. 2012. Mental and physical workload, salivary stress biomarkers and taste perception: Mars desert research station expedition. $N$ Am J Med Sci 4: 577-581.

13) Noel CA, Cassano PA, Dando R. 2017. College-aged males experience attenuated sweet and salty taste with modest weight gain. J Nutr 147: 1885-1891.

14) Basbaum AI, Jessell TM. 2018. Pain. In: Principles of Neural Science (Kandel ER, Schwartz JH, Jessell TM, Siegelbaum SA, Hudspeth AJ, eds), Fifth edition, $p$ 523-545. Medical Sciences International Ltd., Tokyo (in Japanese).

15) Rozin P, Schiller D. 1980. The nature and acquisition of a preference for chili pepper by humans. Motiv Emotion 4: $77-101$.

16) Tominaga M, Tominaga T. 2005. Structure and function of TRPV1. Pflugers Arch Physiol 451: 145-150.

17) Matsumoto T, Miyawaki C, Ue H, Yuasa T, Miyatsuji A, Moritani T. 2000. Effects of capsaicin-containing yellow curry sauce on sympathetic nervous system activity and diet-induced thermogenesis in lean and obese young women. J Nutr Sci Vitaminol 46: 309-315.

18) Bach FW, Yaksh TL. 1995. Release of beta-endorphin immunoreactivity into ventriculo-cisternal perfusate by lumbar intrathecal capsaicin in the rat. Brain Res $\mathbf{7 0 1}$ : 192-200.

19) Kaefer CM, Milner JA. 2008. The role of herbs and spices in cancer prevention. J Nutr Biochem 19: 347-361.

20) Lichtenberger LM, Romero JJ, Carryl OR, Illich PA, Walters ET. 1998. Effect of pepper and bismuth subsalicylate on gastric pain and surface hydrophobicity in the rat. Aliment Pharmacol Ther 12: 483-490.

21) Carstens E, Kuenzler N, Handwerker HO. 1998. Activation of neurons in rat trigeminal subnucleus caudalis by different irritant chemicals applied to oral or ocular mucosa. J Neurophysiol 80: 465-492.

22) Tochikubo O, Oota T, Miyazaki N, Kaneko Y. 1986. A new portable device for 24-hour recording of ambulatory intra-arterial blood pressure and heart rate in hypertensive patients. Jpn Heart J 27: 661-669.

23) Matsumura H, Imai R, Gondo M, Watanabe K. 2012. Evaluation of pain intensity measurement during the removal of wound dressing material using "the PainVision TM system” for quantitative analysis of perception and pain sensation in healthy subjects. Int Wound J 4: $451-455$.

24) France C, Ditto B. 1992. Cardiovascular responses to the combination of caffeine and mental arithmetic, cold pressor, and static exercise stressors. Psychophysiology 29: 272-282.

25) Sawai A, Ohshige K, Yamasue K, Hayashi T, Tochikubo O. 2007. Influence of mental stress on cardiovascular function as evaluated by changes in energy expenditure. Hypertens Res 30: 1019-1027.

26) Minami J, Ishimitsu T, Matsuoka H. 1999. Effects of smoking cessation on blood pressure and heart rate variability in habitual smokers. Hypertension 33: 586-590.

27) Parker MR, Feng D, Chamuris B, Margolskee RF. 2014. Expression and nuclear translocation of glucocorticoid receptors in type 2 taste receptor cells. Neurosci Lett 13: $72-77$.

28) Rentmeister-Bryant H, Green BG. 1997. Perceived irritation during ingestion of capsaicin or piperine: comparison of trigeminal and non-trigeminal areas. Chem Senses 22: $257-266$.

29) Ahmad AH, Zakaria R. 2015. Pain in times of stress. Malays J Med Sci 22: 52-61.

30) McCarty MF, DiNicolantonio JJ, O’Keefe JH. 2015. Capsaicin may have important potential for promoting vascular and metabolic health. Open Heart 2: e000262.

31) Logan H, Lutgendorf S, Rainville P, Sheffield D, Iverson K, Lubaroff D. 2001. Effects of stress and relaxation on capsaicin-induced pain. J Pain 2: 160-170.

32) Mojet J, Christ-Hazelhof E, Heidema J. 2001. Taste perception with age: generic or specific losses in threshold sensitivity to the five basic tastes? Chem Senses 26: 845-860.

33) Ogawa T, Irikawa N, Yanagisawa D, Shiino A, Tooyama I, Shimizu T. 2017. Taste detection and recognition thresholds in Japanese patients with Alzheimer-type dementia. Auris Nasus Larynx 44: 168-173.

34) Ishigaki N, Hirokawa M, Yatani H. 2009. Sex difference in thermal perception and thermal pain thresholds in the trigeminal nerve area. Jpn J Orofacial Pain 2: 15-19.

35) Terkelsen AJ, Andersen OK, Mølgaard H, Hansen J, Jensen TS. 2004. Mental stress inhibits pain perception and heart rate variability but not a nociceptive withdrawal reflex. Acta Physiol Scand 180: 405-414.

36) Kim YK, Hwang JA, Lee HJ, Yoon HK, Ko YH, Lee BH, Jung HY, Hahn SW, Na KS. 2014. Association between norepinephrine transporter gene (SLC6A2) polymorphisms and suicide in patients with major depressive disorder. J Affect Disord 158: 127-132.

37) Tahara T, Shibata T, Nakamura M, Yamashita H, Yoshioka D, Hirata I, Arisawa T. 2010. Homozygous TRPV1 $315 \mathrm{C}$ influences the susceptibility to functional dyspepsia. J Clin Gastroenterol 44: 1-7.

38) Lee SY, Masaoka T, Han HS, Matsuzaki J, Hong MJ, Fukuhara S, Choi HS, Suzuki H. 2016. A prospective study on symptom generation according to spicy food intake and TRPV1 genotypes in functional dyspepsia patients. Neurogastroenterol Motil 19: 1-8. 